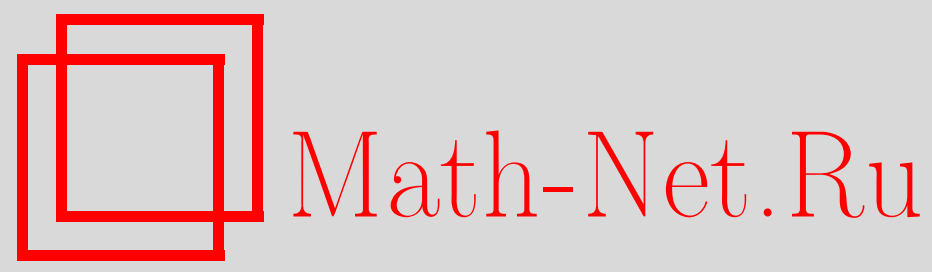

А. М. Райгородский, Вероятностный подход к задаче о дефектах допустимых множеств в решетке, Матем. заметки, 2000, том 68, выпуск 6, 910-916

DOI: https://doi.org/10.4213/mzm1014

Использование Общероссийского математического портала Math-Net.Ru подразумевает, что вы прочитали и согласны с пользовательским соглашением http://www.mathnet.ru/rus/agreement

Параметры загрузки:

IP : 54.89 .56 .158

26 апреля 2023 г., $17: 26: 00$ 


\section{ВЕРОЯТНОСТНЫЙ ПОДХОД К ЗАДАЧЕ О ДЕФЕКТАХ ДОПУСТИМЫХ МНОЖЕСТВ В РЕШЕТКЕ}

\section{А. М. Райгородский}

В настоящей работе для достаточно шшрокого класса множеств получены новые оценки величины дефекта допустимого множества в решетке.

Библиографоия: 16 названий.

1. Введение. Пусть $\Lambda \subset \mathbb{R}^{n}-n$-мерная решетка в евклидовом пространстве, содержашая начало координат $O$. Пусть, кроме того, $\Lambda$ содержит решетку целых чисел $\mathbb{Z}^{n}$ в качестве подрешетки. Рассмотрим репер $\mathscr{E}=O, \boldsymbol{e}_{1}, \ldots, \boldsymbol{e}_{n}$, состоящий из начала координат и из единичных ортов $e_{1}, \ldots, e_{n}$. Понятно, что $\mathscr{E} \subset \Lambda$. Определим величину $f$ как максимальное число, для которого существует некоторая подсистема векторов $\boldsymbol{e}_{i_{1}}, \ldots, \boldsymbol{e}_{i_{f}}$ из репера $\mathscr{E}$, дополнимая до базиса в решетке $\Lambda$. Величину $d(\mathscr{E} ; \Lambda)=n-f$ мы будем назьвать дефектом репера $\mathscr{E}$ относительно решетки $\Lambda$. Иными словами, $d(\mathscr{E} ; \Lambda)$ есть минимальное число векторов, которые необходимо удалить из репера с тем, чтобы оставшаяся система векторов оказалась дополнимой до базиса решетки $\Lambda$.

Рассмотрим теперь произвольную последовательность множеств $\left\{\Omega_{\mathscr{E}}^{n}\right\}_{n=1}^{\infty}, \Omega_{\mathscr{E}}^{n} \subset \mathbb{R}^{n}$, удовлетворяющую следующим условиям.

УСловиЕ 1. Множество $\Omega_{\mathscr{E}}^{n} \subset[-1,1]^{n}$ для всех $n \geqslant 1$.

УСловиЕ 2. Имеет место вложение $\pm \mathscr{E} \subset \Omega_{\mathscr{E}}^{n}$ для всех $n \geqslant 1$.

УСловиЕ 3 . Сечение множества $\Omega_{\mathscr{E}}^{n} \quad k$-мерньм координатным подпространством $\mathscr{R}_{i_{1}, \ldots, i_{k}}$, которое отвечает координатам $x_{i_{1}}, \ldots, x_{i_{k}}$, совпадает с $\Omega_{\mathscr{E}}^{k}$ для всех значений $k \leqslant n$ и $1 \leqslant i_{1}<\cdots<i_{k} \leqslant n$.

Назовем множество $\Omega_{\mathscr{E}}^{n}$ допустимым в решетке $\Lambda$, если $\Omega_{\mathscr{E}}^{n} \cap \Lambda=\left\{O, \pm e_{1}, \ldots, \pm \boldsymbol{e}_{n}\right\}$. В случае, когда множество $\Omega_{\mathscr{E}}^{n}$ допустимо в решетке $\Lambda$, величина дефекта репера $\mathscr{E}$ относительно решетки $\Lambda$ называется дефектом допустимого множества $\Omega_{\mathscr{E}}^{n}$ в решет$\kappa e \Lambda$ и обозначается $d\left(\Omega_{\mathscr{E}}^{n} ; \Lambda\right)$. Определим теперь две величины, зависяшие только от размерности $n$ и рассматриваемого множества $\Omega_{\mathscr{E}}^{n}$ :

$$
d_{n}\left(\Omega_{\mathscr{E}}^{n}\right)=\max _{\Lambda} d\left(\Omega_{\mathscr{E}}^{n} ; \Lambda\right), \quad d_{n}^{*}\left(\Omega_{\mathscr{E}}^{n}\right)=\widetilde{\max _{\Lambda}} d\left(\Omega_{\mathscr{E}}^{n} ; \Lambda\right)
$$

Работа выполнена при финансовой поддержке Российского фонда фундаментальных исследований, гранты № 99-01-00357, № 00-15-96109. 
Здесь в определении величины $d_{n}\left(\Omega_{\mathscr{E}}^{n}\right)$ максимум берется по всем решеткам $\Lambda$, которые удовлетворяют указанньм выше условиям, а в определении величины $d_{n}^{*}\left(\Omega_{\mathscr{E}}^{n}\right)$ предполагается, кроме того, что фактор-групша $\Lambda / \mathbb{Z}^{n}$ циклическая, т.е. найдется вектор $\boldsymbol{a} \in \mathbb{Q}^{n}$ такой, что $\Lambda=\left\langle\mathbb{Z}^{n}, \boldsymbol{a}\right\rangle_{\mathbb{Z}}$.

ПримеР 1. Рассмотрим последовательность множеств $\left\{\Omega_{\mathscr{E}}^{n}\right\}_{n=1}^{\infty}$, каждьй член которой имеет вид

$$
\Omega_{\mathscr{E}}^{n}=\left\{\boldsymbol{x}=\left(x_{1}, \ldots, x_{n}\right) \in \mathbb{R}^{n}:\left|x_{1}\right|^{\alpha}+\cdots+\left|x_{n}\right|^{\alpha} \leqslant 1\right\} .
$$

(Здесь $\alpha>0$ - некоторая произвольная константа.) Ясно, что для такой последовательности множеств вьполнены все условия 1-3. Заметим, что, в частности, при $\alpha=2$ множество $\Omega_{\mathscr{E}}^{n}=\mathscr{B}_{\mathscr{E}}^{n}$ есть единичный шар, а при $\alpha=1$ множество $\Omega_{\mathscr{E}}^{n}=\mathscr{O}_{\mathscr{E}}^{n}$ есть единичньй октаэдр. Эти два частных случая были рассмотрены в работах [1] и [2], где для величин $d_{n}\left(\mathscr{B}_{\mathscr{E}}^{n}\right), d_{n}\left(\mathscr{O}_{\mathscr{E}}^{n}\right), d_{n}^{*}\left(\mathscr{B}_{\mathscr{E}}^{n}\right)$ и $d_{n}^{*}\left(\mathscr{O}_{\mathscr{E}}^{n}\right)$ были получены точные по порядку оценки. Отметим также, что для случая малых размерностей сходные задачи рассматривались в работах [3]-[8].

ПРИмеР 2. Рассмотрим последовательность множеств $\left\{\Omega_{\mathscr{E}}^{n}\right\}_{n=1}^{\infty}$ такую, что

$$
\Omega_{\mathscr{E}}^{n}=\left\{\boldsymbol{x}=\left(x_{1}, \ldots, x_{n}\right) \in \mathbb{R}^{n}: N^{n-1} \prod_{i=1}^{n} \max \left\{\frac{1}{N},\left|x_{i}\right|\right\} \leqslant 1\right\} .
$$

(Здесь мы считаем, что $N \in \mathbb{N}$ и $N \geqslant 2$.) Таким образом, $\left\{\Omega_{\mathscr{E}}^{n}\right\}_{n=1}^{\infty}$ - это последовательность так называемых $n$-мерных гиперболических крестов. Нетрудно видеть, что и в этом случае вьполнены условия $1-3$.

Основной целью настоящей работы является получение нижних оценок величины $d_{n}^{*}\left(\Omega_{\mathscr{E}}^{n}\right)$ для достаточно широкого класса последовательностей множеств $\left\{\Omega_{\mathscr{E}}^{n}\right\}_{n=1}^{\infty}$. Тем самым результаты статьи обобщают результаты работы [1]. Заметим, что в работе [9] были также получены некоторые обобщения результатов работы [1]. При этом если в [9] доказательства соответсвуюших теорем существенно опирались на некоторую эффективную вспомогательную конструкцию, то в настоящей работе доказательства результатов будут носить чисто вероятностньй характер, что позволит независимым образом построить обобщения, принципиально отличные от ранее предложенных. Так существенную роль при оценке величины $d_{n}^{*}\left(\Omega_{\mathscr{E}}^{n}\right)$ будут играть подсчеты средних значений, возникающих в задаче о покрытии (см. [10]-[13]) и в геометрии чисел (см. теорему Минковского-Главки в [14], [15], а также [16]).

2. Формулировки результатов. Из результатов, полученных в работах [1] и [2], вытекает следующая

Tеорема 1. Существуют абсолютные постоянные $c_{1}>0, c_{2}>0 u c_{3}>0$ такие, что имеют место оценки:

$$
\begin{gathered}
\max \left\{d_{n}^{*}\left(\mathscr{O}_{\mathscr{E}}^{n}\right), d_{n}^{*}\left(\mathscr{B}_{\mathscr{E}}^{n}\right)\right\} \leqslant \frac{c_{1} n(\log \log n)^{2}}{\log n} \\
\min \left\{d_{n}^{*}\left(\mathscr{O}_{\mathscr{E}}^{n}\right), d_{n}^{*}\left(\mathscr{B}_{\mathscr{E}}^{n}\right)\right\} \geqslant \frac{c_{2} n(\log \log n)^{2}}{\log n}, \quad \min \left\{d_{n}\left(\mathscr{O}_{\mathscr{E}}^{n}\right), d_{n}\left(\mathscr{B}_{\mathscr{E}}^{n}\right)\right\} \geqslant n-\frac{c_{3} n}{\log n}
\end{gathered}
$$


В недавно выполненной работе автора [9] при некоторых достаточно сложных ограничениях, наложенньх на последовательность множеств $\left\{\Omega_{\mathscr{E}}^{n}\right\}_{n=1}^{\infty}$, были получены верхние и нижние оценки величины $d_{n}^{*}\left(\Omega_{\mathscr{E}}^{n}\right)$. При этом каждой последовательности множеств $\left\{\Omega_{\mathscr{E}}^{n}\right\}_{n=1}^{\infty}$ ставились в соответствие два набора параметров: с одной стороны, определялся набор параметров $k, n=n(k)$ (размерность) и $f$, причем величина $f$ строилась алгоритмически в соответствии с некоторыми комбинаторньми условиями, а с другой стороны, рассматривались параметры $n^{\prime}$ (размерность), $k^{\prime}$ и $s^{\prime}$ (подробности см. в [9]). С использованием этих параметров и некоторых ограничений, наложенных на них, была доказана

Теорема 2. Имеют место неравенства:

$$
d_{n}^{*}\left(\Omega_{\mathscr{E}}^{n}\right) \gg \frac{n}{k} \log (f+1), \quad d_{n^{\prime}}^{*}\left(\Omega_{\mathscr{E}}^{n^{\prime}}\right) \ll \frac{n^{\prime}}{k^{\prime}} \log \frac{s^{\prime} k^{\prime}}{n^{\prime}} .
$$

(Здесь последнее неравенство выполнено только для выпуклых О-симметричных множеств.)

В частности, в [9] было показано, что результаты теоремы 1 могут быть получены в качестве следствия из теоремы 2 при $\Omega_{\mathscr{E}}^{n}=\mathscr{O}_{\mathscr{E}}^{n}$ и при $\Omega_{\mathscr{E}}^{n}=\mathscr{B}_{\mathscr{E}}^{n}$ соответственно.

Отметим также, что значение величины $d_{4}\left(\mathscr{O}_{\mathscr{E}}^{n}\right)$ было найдено в $[4]$, величина $d_{5}\left(\mathscr{O}_{\mathscr{E}}^{n}\right)$ была подсчитана в [6], а результаты, касаюшиеся определения величины $d_{n}\left(\mathscr{B}_{\mathscr{E}}^{n}\right)$ при $n \leqslant 8$, следуют из [7] и [8].

Перейдем теперь непосредственно к формулировке основного результата настоящей работы. Для этого нам потребуется ввести некоторые вспомогательные параметры. Зафиксируем произвольную последовательность множеств $\left\{\Omega_{\mathscr{E}}^{n}\right\}_{n=1}^{\infty}$, удовлетворяющую условиям 1-3. Предположим, что этой последовательности множеств можно сопоставить некоторую последовательность $\{v(n)\}_{n=1}^{\infty}$ положительных действительных чисел, обладающую следующими свойствами.

Свойство 1. Функция $v(n)$ строго монотонно возрастает, причем $v(n) \geqslant n$ при $n \geqslant n_{0}$.

Свойство 2. Найдется абсолютная постоянная $c>0$ такая, что для всякого достаточно большого натурального числа $k$, для величины $n=\left[e^{v(k) / c}\right]$ и для натурального $s$ такого, что $v(n) /(c \log n) \ll s \leqslant v(n) /(c \log n)$, выполнено неравенство

$$
\left(\operatorname{card}\left(\Omega_{\mathscr{E}}^{t} \cap \Lambda_{m_{1}, \ldots, m_{t}}\right)\right)\left(\frac{n+s}{n-1}\right)^{t} e^{v(t) / c}<\frac{1}{n^{3}}\left(\operatorname{det} \Lambda_{m_{1}, \ldots, m_{t}}\right)^{-1}
$$

(Здесь $k \leqslant t \leqslant n$, a $\Lambda_{m_{1}, \ldots, m_{t}}$-это произвольная решетка вида

$$
\Lambda_{m_{1}, \ldots, m_{t}}=\left\langle\left(\frac{1}{m_{1}}, 0, \ldots, 0\right), \ldots,\left(0, \ldots, 0, \frac{1}{m_{t}}\right)\right\rangle_{\mathbb{Z}}
$$

где, в свою очередь, $m_{i} \in \mathbb{N}$ и $m_{i} \geqslant n$ для всех $i=1, \ldots, t$.) 
Свойство 3. Пусть $c, k, n$ и $s$ такие же, как в свойстве 2. Тогда

$$
\frac{1}{C_{C_{n}^{k}}^{s}} \sum_{t=k+1}^{n} C_{n}^{t} \sum_{i=0}^{r_{t}} C_{C_{t}^{k}}^{[v(t) /(c \log n)]+1+i} C_{C_{n}^{k}-C_{t}^{k}}^{s-[v(t) /(c \log n)]-1-i} \rightarrow 0, \quad k \rightarrow \infty .
$$

Здесь $r_{t}=s-[v(t) /(c \log n)]-1$.

Свойство 4. Те же параметры $c, k, n$ и $s$ удовлетворяют следующим асимптотическим соотношениям:

$$
n \rightarrow \infty, \quad s \rightarrow \infty, \quad \log \frac{s k}{n} \rightarrow \infty \text { при } k \rightarrow \infty, \quad \log \frac{s k}{n}=o(k) .
$$

Имеет место следующая

ТЕОРемА 3. Для произвольной последовательности мнохсеств $\left\{\Omega_{\mathscr{E}}^{n}\right\}_{n=1}^{\infty}$, удовлетворяющей условиям 1-3, и произвольной последовательности положительных чисел $\{v(n)\}_{n=1}^{\infty}$, удовлетворяющей свойствам 1-4, имеет место асимптотическая оценка

$$
d_{n(k)}^{*}\left(\Omega_{\mathscr{E}}^{n(k)}\right) \gg \frac{n(k)}{k} \log \frac{s(k) k}{n(k)}, \quad k \rightarrow \infty .
$$

Заметим, что в случае, когда всякое множество $\Omega_{\mathscr{E}}^{n}$ из рассматриваемой последовательности измеримо по Жордану и $V\left(\Omega_{\mathscr{E}}^{n}\right)$ - его объем, в качестве фигурирующей в условиях теоремы 3 функции $v(n)$ часто бывает удобно брать величину $v(n)=$ $-\log V\left(\Omega_{\mathscr{E}}^{n}\right)$. Отметим, кроме того, еще раз, что в то время, как доказательство теоремы 2 носит в существенной степени конструктивный характер, доказательство теоремы 3 полностью вероятностное. Заметим также, что нетрудно привести пример, когда работает теорема 2 , а теорема 3 неприменима. А именно, применяя теорему 2 к последовательности гиперболических крестов $\left\{\Omega_{\mathscr{E}}^{n}\right\}_{n=1}^{\infty}$ с достаточно большим $N$ (см. пример 2), можно получить оценку $d_{n}^{*}\left(\Omega_{\mathscr{E}}^{n}\right) \gg n / \log n$. При этом нетрудно видеть, что к такой последовательности теорема 3 неприменима. С другой стороны, задача проверки условий теоремы 3 значительно менее трудоемкая, чем задача проверки условий теоремы 2. Тем самьм, основным преимуществом теоремы 2 является ее конструктивность, в то время как в теореме 3 условия являются более компактньми, а доказательство менее сложным.

Для доказательства теоремы 3 нам потребуется некоторая комбинаторная лемма.

3. Комбинаторная лемма. Пусть $\mathfrak{R}_{n}=\{1, \ldots, n\}$ - множество, состоящее из $n$ элементов. Обозначим через $\mathscr{M}=\left\{M_{1}, \ldots, M_{s}\right\} \subset 2^{\Re_{n}}$ произвольную совокупность подмножеств $M_{j}, j=1, \ldots, s$, множества $\mathfrak{R}_{n}$. Подмножество $S \subset \mathfrak{R}_{n}$ назьвается системой общих представителей (с.о.п.) для совокупности множеств $\mathscr{M}$, если $S \cap M_{j} \neq \varnothing$ для всех $j=1, \ldots, s$. Положим $\tau=\tau(\mathscr{M})=\min _{S} \operatorname{card} S=\operatorname{card} \Sigma$. (Здесь минимум берется по всем возможным с.о.п. $S$ для совокупности множеств $\mathscr{M}$.) Множество $\Sigma$, определяющееся по заданной совокупности $\mathscr{M}$, вообще говоря, неоднозначно, естественно назьвать наименьшей с. о.n. для $\mathscr{M}$. Максимальным $\tau$ мы назовем величину $Z(n, k, s)=\max \mathscr{M} \tau(\mathscr{M})$, где максимум берется по всем совокупностям множеств $\mathscr{M}=\left\{M_{1}, \ldots, M_{s}\right\} \subset 2^{\Re_{n}}$ таким, что $\operatorname{card} M_{j} \geqslant k, j=1, \ldots, s$. Различные 
оценки и асимптотические формулы для величины $Z(n, k, s)$ получены, в частности, в работах [11] и [12] (см. также имеющиеся в этих работах ссылки).

Пусть $c>0$ - некоторая произвольная постоянная, а $v(n)$ - некоторая строго монотонно возрастающая функция такая, что $v(n) \geqslant n$ при $n \geqslant n_{0}$. Тогда совокупность множеств $\mathscr{M} \subset 2^{\Re_{n}}$ назьвается равномерно расположенной (р.p.) в $\Re_{n}$ с функцией $v(n)$ и константой $c$, если для любого $t$ такого, что $1 \leqslant t \leqslant n$, и для всякого множества $R \subset \mathfrak{R}_{n}$, $\operatorname{card} R=t$, вьполнено неравенство

$$
\operatorname{card}\{M \in \mathscr{M}: M \subset R\} \leqslant \frac{v(t)}{c \log n} .
$$

Пусть параметры $c, k, n, s$ и $v(n)$ удовлетворяют всем условиям теоремы 3 . Тогда имеет место следуюшая

Лемма. Существует совокупность множеств $\mathscr{M}=\left\{M_{1}, \ldots, M_{s}\right\} \subset 2^{\Re_{n}}$ maкая, что выполняются следуюшие условия:

1) $\operatorname{card} M_{j}=k$ для всех $j=1, \ldots, s$;

2) $\mathscr{M}$ р.р. в $\Re_{n}$ с функцией $v(n)$ и константой $c$;

3) $\tau(\mathscr{M}) \gg \frac{n}{k} \log \frac{s k}{n}$.

ДокАЗАТЕЛЬСТво. Предлагаемое доказательство мы проведем с помощью процедуры усреднения. Иньми словами, все дальнейшие рассуждения будут основаны на том хорошо известном факте, что если на некотором множестве $\mathscr{F}(m)$ задан неотрицательный целочисленный параметр $\delta(f)$ и

$$
S(m)=\frac{1}{\operatorname{card} \mathscr{F}(m)} \sum_{f \in \mathscr{F}(m)} \delta(f) \rightarrow 0 \quad \text { при } m \rightarrow \infty
$$

то $\delta(f)=0$ для почти всех элементов $f \in \mathscr{F}(m)$.

Обозначим через $\mathfrak{M}$ класс всех возможных совокупностей множеств $\mathscr{M}=\left\{M_{1}, \ldots\right.$, $\left.M_{s}\right\} \subset 2^{\mathfrak{R}_{n}}$ таких, что card $M_{j}=k$ для всех $j=1, \ldots, s$. Ясно, что card $\mathfrak{M}=C_{C_{n}^{k}}^{s}$. Покажем сначала, что почти все элементы класса $\mathfrak{M}$ p.p. в $\mathfrak{R}_{n}$ с функцией $v(n)$ и константой $c$. С этой целью каждому натуральному числу $t, k+1 \leqslant t \leqslant n$, и каждому множеству $R \subset \mathfrak{R}_{n}, \operatorname{card} R=t$, сопоставим параметр $\delta_{t, R}(\mathscr{M})$, определенный на классе $\mathfrak{M}$ следующим образом:

$$
\delta_{t, R}(\mathscr{M})=\left\{\begin{array}{l}
0, \text { если } \operatorname{card}\{M \in \mathscr{M}: M \subset R\} \leqslant \frac{v(t)}{c \log n} \\
1, \text { если } \operatorname{card}\{M \in \mathscr{M}: M \subset R\}>\frac{v(t)}{c \log n}
\end{array}\right.
$$

Положим

$$
\delta(\mathscr{M})=\sum_{t=k+1}^{n} \sum_{R \subset \mathfrak{R}_{n}: \operatorname{card} R=t} \delta_{t, R}(\mathscr{M}) .
$$


Тогда нетрудно видеть, что равномерная расположенность некоторой совокупности множеств $\mathscr{M}$ равносильна тому, что $\delta(\mathscr{M})=0$. С другой стороны, легко проверить, что свойство 3 влечет следующее асимптотическое выражение:

$$
\frac{1}{C_{C_{n}^{k}}^{s}} \sum_{\mathscr{M} \in \mathfrak{M}} \delta(\mathscr{M}) \rightarrow 0 \quad \text { при } k \rightarrow \infty .
$$

Отсюда же немедленно следует, что почти все совокупности множеств $\mathscr{M} \in \mathfrak{M}$ р.p. в $\mathfrak{R}_{n}$ с функцией $v(n)$ и константой $c$. Иначе говоря, мы доказали, что почти для всех совокупностей из рассматриваемого класса вьполнено условие 2) леммы. Тем самым для завершения доказательства нам достаточно проверить, что условие 3) леммы также выполнено для почти всех совокупностей из класса $\mathfrak{M}$. В самом деле, применяя теорему 13 из работы [11] с учетом свойств 1 и 4, мы получаем требуемое утверждение. Лемма доказана.

Заметим, что в [9] была также доказана аналогичная лемма, но там построение совокупности множеств $\mathscr{M}$, обладающей похожими свойствами, носило эффективньй характер, тогда как здесь мы имеем дело исключительно с подсчетом средних значений.

4. Доказательство теоремы 3. Перейдем непосредственно к доказательству теоремы 3 . Мы утверждаем, что найдется вектор $\boldsymbol{a} \in \mathbb{Q}^{n}$ такой, что множество $\Omega_{\mathscr{E}}^{n}$ допустимо в решетке $\Lambda_{\boldsymbol{a}}=\left\langle\mathbb{Z}^{n}, \boldsymbol{a}\right\rangle_{\mathbb{Z}}$ и

$$
d\left(\Omega_{\mathscr{E}}^{n} ; \Lambda_{\boldsymbol{a}}\right) \gg \frac{n}{k} \log \frac{s k}{n}
$$

Пусть $\mathscr{M}$ - совокупность множеств, построенная в лемме. С помощью стандартной процедуры (см. [1] и [9]) поставим этой совокупности в соответствие семейство $n$-мерньх рациональных векторов V. В работе [1] было доказано

ПРЕДЛОЖЕНИЕ. ДЛя всякого вектора $\boldsymbol{a} \in \mathbf{V}$ выполнено равенство

$$
d\left(\mathscr{E} ; \Lambda_{\boldsymbol{a}}\right)=\tau(\mathscr{M})
$$

Из предложения следует оценка

$$
d\left(\mathscr{E} ; \Lambda_{\boldsymbol{a}}\right) \gg \frac{n}{k} \log \frac{s k}{n}
$$

для всех $\boldsymbol{a} \in \mathbf{V}$, так что для завершения доказательства теоремы 3 достаточно выделить из семейства векторов $\mathbf{V}$ хотя бы один рациональньй вектор $\boldsymbol{a}$, добавление которого к решетке целых чисел задает новую решетку $\Lambda_{\boldsymbol{a}}$, в которой допустимо множество $\Omega_{\mathscr{E}}^{n}$. Выделение такого вектора также может быть осуществлено с помощью процедуры усреднения с учетом условий 1-3 и свойства 2. Такая процедура подробно описана в [1] для $\Omega_{\mathscr{E}}^{n}=\mathscr{O}_{\mathscr{E}}^{n}$ и в $[9]$ для общего случая, поэтому в настоящей работе мы ее не проводим. Теорема доказана. 


\section{СПИСОК ЦИТИРОВАННОЙ ЛИТЕРАТУРЫ}

[1] Райгородский А. М. Дефект допустимых шаров и октаэдров в решетке и системы общих представителей // Матем. сб. 1998. Т. 189. №6. С. 117-141.

[2] Мощевитин Н. Г. Дефект допустимого октаэдра в решетке // Матем. заметки. 1995. Т. 58. C. $558-568$.

[3] Cusick T.W., Wolfskill J. Lattice octahedra and sums of powers of linear forms // J. London Math. Soc. (2). 1988. V. 38. P. 207-215.

[4] Mordell L. J. Lattice octahedra // Canad. J. Math. 1960. V. 12. P. 297-302.

[5] Bantegnie R. Le problème des octaedres en dimension 5 // Acta Arith. 1968. V. 14. P. 185-202.

[6] Wessels U. Die Sätze von White und Mordell über kritische Gitter von Polytopen in den Dimensionen 4 und 5. Diplomarbeit: Mathematisches Institut der Ruhr-Universität Bochum, 1989.

[7] Рышков С. С. К проблеме отыскания совершенных квадратичных форм от многих переменных // Тр. МИАН. 1976. Т. 142. С. 215-239.

[8] Захарова Н. В. Центрировки 8-мерных решеток, сохраняющие репер последовательных минимумов // Тр. МИАН. 1980. Т. 152. С. 97-123.

[9] Raigorodskii A. M. The defects of admissible sets in a lattice, and systems of common representatives // Grazer Mathematische Berichte. 1999. № 338. P. 31-62.

[10] Эрдеш П., Спенсер Дж. Вероятностные методы в комбинаторике. М.: Мир, 1976.

[11] Кузюрин Н. Н. Асимптотическое исследование задачи о покрытии // Проблемы кибернетики. 1980. №37. С. 19-56.

[12] Райгородский А. М. Системы общих представителей // Фундамент. и прикл. матем. 1999. T. 5. № 3. C. $851-860$.

[13] Тараканов В. Е. Комбинаторные задачи и $(0,1)$-матрицы. М.: Физматлит, 1985.

[14] Gruber P. M., Lekkerkerker C. G. Geometry of Numbers. Amsterdam: North-Holland, 1987.

[15] Cassels J. W. S. An Introduction to the Geometry of Numbers. Berlin-Göttingen-Heidelberg: Springer-Verlag, 1959.

[16] Коробов Н. М. Теоретико-числовые методы в приближенном анализе. Физматлит: М., 1963. 